\title{
Diagnóstico por imagem de arteriopatia cerebral associada a mutação ACTA2: relato de caso
}

\section{Diagnostic imaging of cerebral arteriopathy associated with ACTA2 mutation: case report}

\author{
Valéria Cristina do Rosário Rebouças Rocha ${ }^{1}$. Thais de Maria Frota Vasconcelos ${ }^{1}$. Clarissa Pinto de \\ Albuquerque $^{1,2}$. Paulo Ribeiro Nóbrega ${ }^{1,2}$. José Daniel Vieira de Castro ${ }^{1}$. \\ 1 Universidade Federal do Ceará (UFC), Fortaleza, Ceará, Brasil. 2 Hospital Universitário Walter Cantídio (HUWC), Fortaleza, \\ Ceará, Brasil.
}

\section{RESUMO}

Objetivos: descrever as características radiológicas que permitem o reconhecimento da arteriopatia cerebral associada à mutação ACTA2 nas imagens de ressonância magnética. Metodologia: foram analisadas imagens de ressonância magnética (RM) e angiorresonância (ARM) de crânio. Resultados: paciente do sexo feminino, 19 anos, com déficit cognitivo e cefaleia occipital, evoluindo com paraparesia à direita e amaurose bilateral. Realizou RM e ARM de crânio, que demonstrou achados característicos: retificação das estruturas arteriais, hipoplasia e arqueamento do corpo caloso anteriormente, aspecto em "V" do joelho do corpo caloso e proeminente impressão da artéria basilar sobre a ponte. Foram também vistos focos de alteração de sinal em zona de fronteira vascular frontal direita. Conclusão: Mutações ACTA2 são raras e caracterizam-se por uma disfunção sistêmica do músculo liso, causando alterações cerebrovasculares, dissecções e aneurismas de aorta, hipertensão pulmonar, dentre outros. A arteriopatia cerebral é responsável por episódios de acidente vascular cerebral na infância, sendo caracterizada por retificação das estruturas arteriais e oclusão progressiva das artérias carótidas internas terminais e seus ramos. Tem características de imagem únicas, que permitem diferenciá-la da doença Moya-Moya, conforme acima descritas. Os radiologistas devem, portanto, reconhecer tais achados uma vez que o correto diagnóstico tem importantes implicações no manejo e no prognóstico.

Palavras-chave: Vasculite. Mutação. Transtornos cerebrovasculares. Angiografia por ressonância magnética.

\section{ABSTRACT}

Objectives: To describe the radiological characteristics that allow the recognition of cerebral arteriopathy cssociated with ACTA2 mutation in magnetic resonance imaging. Methodology: Magnetic resonance (MRI) and magnetic resonance angiography of the brain (MRA) images were analyzed. Results: Female patient, 19 years old, with cognitive deficit and occipital headache, evolving with paraparesis on the right and bilateral amaurosis. She had brain MRI and MRA performed, which showed characteristic findings: arterial structures rectification, hypoplasia and anterior bending of the corpus callosum, "V" aspect of the corpus callosum genu and prominent impression of the basilar artery over the pons. Signal alteration foci were also seen in the right frontal vascular border area. Conclusion: ACTA2 mutations are rare and are characterized by systemic smooth muscle dysfunction, causing cerebrovascular changes, aortic dissections and aneurysms, pulmonary hypertension, among others. Cerebral arteriopathy is responsible for episodes of stroke in childhood, being characterized by rectification of arterial structures and progressive occlusion of the terminal internal carotid arteries and their branches. It has unique image characteristics, which differentiate it from MoyaMoya disease, as described above. Radiologists must, therefore, recognize these findings since the correct diagnosis has important implications for management and prognosis.

Keywords: Vasculitis. Mutation. Cerebrovascular disorders. Magnetic resonance angiography.

Autor correspondente: Valéria Cristina do Rosário Rebouças Rocha, Rua Júlio Siqueira, 632, Dionísio Torres, Fortaleza, Ceará. CEP: 60.130-090. Telefone: +55 85 98681-0604. E-mail: valeriacrr@hotmail.com

Conflito de interesses: Não há qualquer conflito de interesses por parte de qualquer um dos autores.

Recebido em: 03 Fev 2021; Revisado em: 11 Jun 2021; Aceito em: 03 Nov 2021. 


\section{INTRODUÇÃO}

A mutação do gene ACTA2 é responsável por uma síndrome de disfunção multissistêmica do músculo liso, que predispõe a acidentes vasculares cerebrais isquêmicos de início precoce, tendo uma aparência característica nas imagens cerebrovasculares. O gene ACTA2 codifica uma proteína específica do músculo liso vascular, chamada $\alpha$-actina. Pacientes com mutação nesse gene apresentam desorganização das células musculares lisas da parede dos vasos, causando sua disfunção.

As mutações heterozigóticas desse gene podem resultar em uma variedade de doenças vasculares sistêmicas além de acidentes vasculares cerebrais, como doença arterial coronariana também de início precoce, doença de Moyamoya e aneurismas da aorta torácica com dissecção. ${ }^{1}$ A arteriopatia cerebral nessa condição apresenta-se, tipicamente, com eventos isquêmicos iniciados na faixa etária pediátrica ou adolescência, com sequelas neurológicas iniciadas já na infância.

O objetivo deste trabalho é destacar a importância do reconhecimento do aspecto neurorradiológico na arteriopatia cerebral associada à mutação ACTA2, para o correto diagnóstico, manejo clínico e aconselhamento genético dessa condição.

\section{APRESENTAÇÃO DO CASO}

Paciente feminina de 19 anos, com história de déficit cognitivo, iniciou cefaleia de forte intensidade em região occipital associada a vômitos persistentes, seguidos de redução da força no dimídio direito. Os sintomas melhoraram espontaneamente, quando, após três meses, apresentou turvação visual que evoluiu em poucos dias para amaurose bilateral. Internou para investigação dos sintomas, sendo realizada ressonância de crânio, que evidenciou alterações morfológicas no corpo caloso, caracterizadas por hipoplasia do rostro do corpo caloso (Figuras 1 e 2), além de aparência em "V" no corte axial da sua porção mais anterior (Figura 3).

Figura 1. Imagem de ressonância magnética em aquisição sagital ponderada em T2.

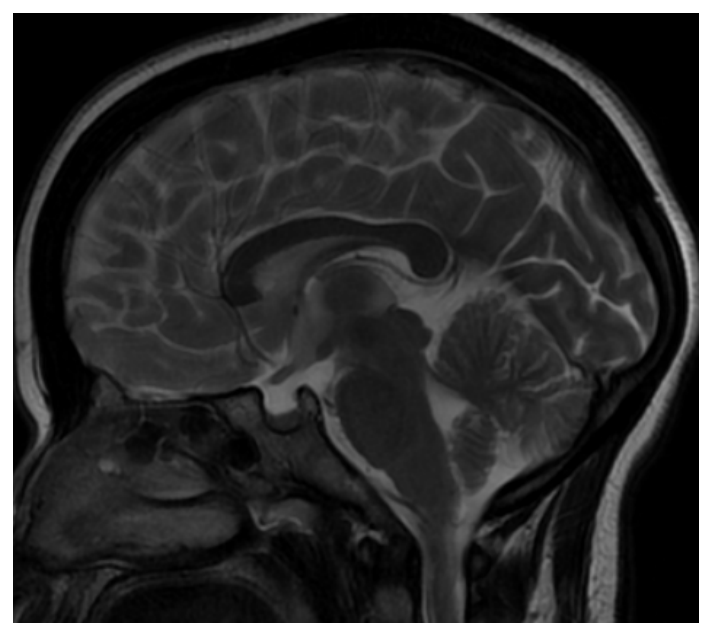

Nota: hipoplasia típica e curvatura anterior do corpo caloso, com giro do cíngulo anterior deficiente.
Figura 2. Imagem de ressonância magnética em aquisição sagital ponderada em T1.

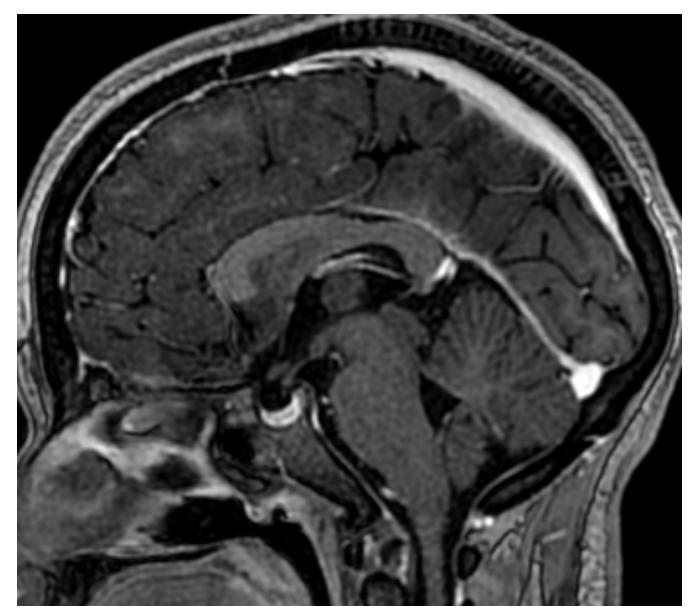

Nota: hipoplasia típica e curvatura anterior do corpo caloso, com giro do cíngulo anterior deficiente.

Figura 3. Imagem de ressonância magnética T1 spgr axial.

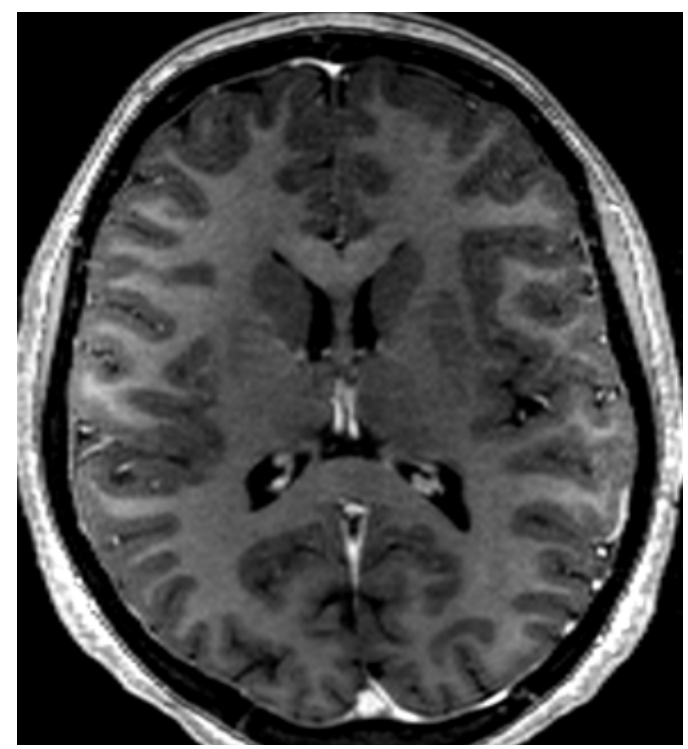

Nota: aspecto em "V" característico da porção anterior do corpo caloso.

Evidenciou, ainda, impressão da artéria basilar sobre a superfície anterior da ponte com esboço de duas proeminências laterais simétricas ("twin-peaks pons") (Figura 4). Prosseguiu-se investigação diagnóstica com angiorressonância de crânio, que evidenciou anormalidades difusas das artérias intracranianas, caracterizadas por estreitamento e retificação dos vasos intracranianos, bem como dilatação das artérias carótidas internas extradurais (Figuras 5 e 6). Uma ampla investigação das causas da vasculite do sistema nervoso central foi realizada, porém, nenhuma etiologia infecciosa, autoimune ou inflamatória foi identificada. A genotipagem ACTA2 não foi realizada no caso em questão devido a sua indisponibilidade no nosso serviço. 
Figura 4. Imagem de ressonância magnética axial ponderada em T2 ao nível da ponte.

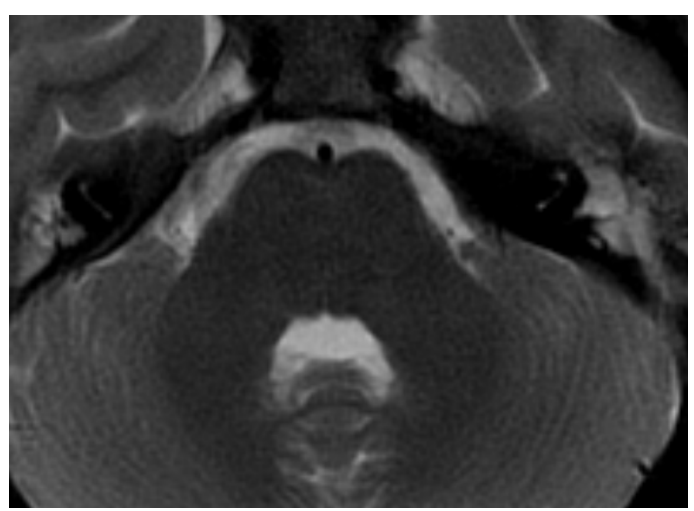

Nota: sinal da "twin-peaks pons": caracterizado por uma impressão da artéria basilar na superfície anterior da ponte com consequente redução do seu diâmetro anterioposterior, gerando duas proeminências simétricas.

Figura 5. Imagem de angiorressonância magnética do crânio MIP sagital

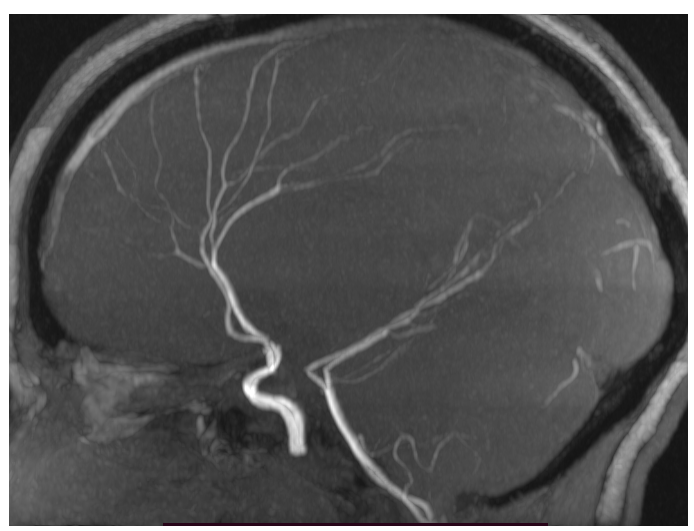

Nota: retificação do trajeto e redução do calibre da maioria das artérias intra-cranianas, sem evidência de circulação colateral.

Figura 6. Imagem de angiorressonância magnética do crânio MIP coronal.

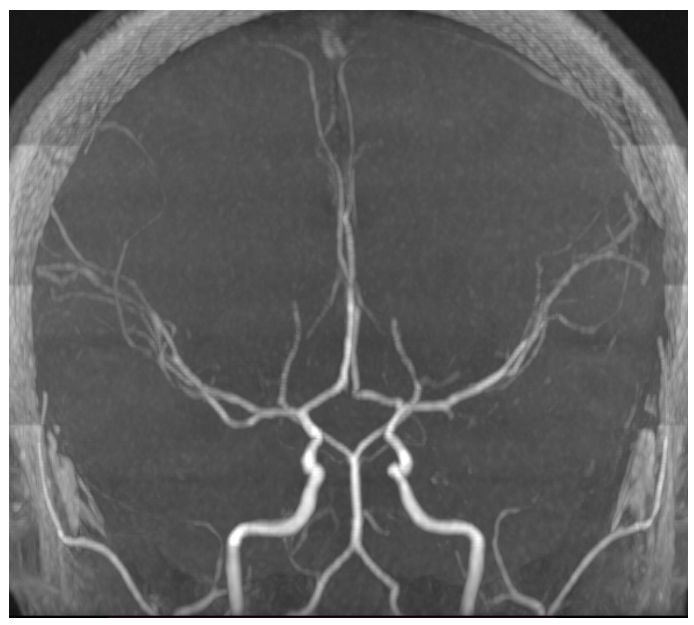

Nota: retificação do trajeto e redução do calibre da maioria das artérias intra-cranianas, sem evidência de circulação colateral.

\section{DISCUSSÃO E CONCLUSÃO}

O diagnóstico diferencial de uma doença cerebrovascular difusa inclui etiologias genéticas, autoimunes e infecciosas. A caracterização das alterações vasculares permite o reconhecimento de padrões que podem estreitar o diagnóstico.

A arteriopatia cerebral secundária à mutação ACTA2 é uma doença cerebrovascular recentemente descrita, observada em pacientes com a chamada síndrome de disfunção multissistêmica do músculo liso, apresentandose tipicamente com eventos isquêmicos na faixa etária pediátrica. A mutação ACTA2 é responsável por uma disfunção sistêmica dos músculos lisos, resultando em doença oclusiva secundária à fibrose e espessamento da parede vascular.

As características dessa condição nas imagens cranianas incluem circulação carotídea interna aberrante com dilatação dos segmentos proximais, doença oclusiva nos segmentos distais e dolicoectasia das artérias carótidas internas proximais. A angioressonância costuma evidenciar dilatação fusiforme dos segmentos cavernosos e clinoide da artéria carótida interna, estenose do segmento terminal da carótida interna frequentemente estendendo-se para a artéria cerebral média e segmento pré-comunicante da artéria cerebral anterior, e ausência de colaterais basais "moyamoya". ${ }^{2}$

As principais características que distinguem a arteriopatia cerebral secundária à mutação ACTA2 da arteriopatia Moyamoya são a ectasia das artérias carótidas internas proximais, um acometimento mais difuso da arteriopatia, com retificação das estruturas arteriais, bem como a ausência de circulação colateral lenticuloestriada.

A aparência característica dos picos simétricos na ponte observada no plano axial (twin-peaks pons) é decorrente da indentação da superfície pontina causada pela impressão da artéria basilar. ${ }^{3}$ Pode haver também irregularidade das superfícies laterais da ponte, pela impressão das artérias pontinas provenientes da artéria basilar, não presente no presente caso.

Acredita-se que o aumento da rigidez das artérias intracranianas resultaria tanto no curso retificado característico dos vasos nessa condição, quanto nas alterações morfológicas cerebrais descritas, como consequência do efeito mecânico local desses vasos. $^{4}$

As anomalias do desenvolvimento cerebral incluem displasia do corpo caloso, com redução do joelho e ausência do rostro, orientação anormal do giro do cíngulo, podendo haver também sulcação anômala nos lobos frontais.

A paciente do caso relatado apresentou os achados de neuroimagem que favoreceram o diagnóstico da arteriopatia cerebral secundária à mutação ACTA2. Tais características permitem a suspeição diagnóstica. É sabido que o diagnóstico definitivo requer a realização da genotipagem. Entretanto, 
este é um teste frequentemente não disponível e não acessível em serviços públicos, tornando fundamental o diagnóstico presuntivo através do quadro clínico e do reconhecimento dos achados de neuroimagem característicos.

\section{REFERÊNCIAS}

1. Guo DC, Papke CL, Tran-Fadulu V, Regalado ES, Avidan N, Johnson RJ, et al. Mutations in smooth muscle alpha-actin (ACTA2) cause coronary artery disease, stroke, and Moyamoya disease, along with thoracic aortic disease. Am J Hum Genet. 2009;84(5):617-27. doi: 10.1016/j.ajhg.2009.04.007.

2. Amans MR, Stout C, Fox C, Narvid J, Hetts SW, Cooke DL, et al. Cerebral arteriopathy associated with Arg179His ACTA2 mutation. BMJ Case Reports. 2013;2013: bcr2013010997. doi.org/10.1136/ bcr-2013-010997

\section{Aprovação ética e consentimento para participar}

Este trabalho foi submetido à Plataforma Brasil, com aprovação do Comitê de Ética em Pesquisa do Hospital Universitário Walter Cantídio (HUWC-UFC), com número do parecer 4.564.597. O consentimento para participação neste relato de caso foi obtido do sujeito investigado incluído no relato.

3. D'Arco F, Alves CA, Raybaud C, Chong WKK, Ishak GE, Ramji S, et al. Expanding the Distinctive Neuroimaging Phenotype of ACTA2 Mutations. AJNR Am J Neuroradiol . 2018 Nov;39(11):2126-2131. doi: 10.3174/ajnr.A5823

4. Georgescu MM, Pinho MC, Richardson TE, Torrealba J, Buja LM, Milewicz DM, et al. The defining pathology of the new clinical and histopathologic entity ACTA2-related cerebrovascular disease. Acta Neuropathol Commun. 2015;3:81.

\section{Como citar:}

Rocha VC, Vasconcelos TM, Albuquerque CP, Nóbrega PR, Castro JD. Diagnóstico por imagem de arteriopatia cerebral associada a mutação ACTA2: relato de caso. Rev Med UFC. 2022;62(1):1-4. 